\title{
Giant magnetooscillations of the Josephson current
}

\author{
L. P. Gor'kov \\ National High Magnetic Field Laboratory, Florida State University, Tallahassee, FL 32306, USA and \\ L. D. Landau Institute for Theoretical Physics, Russia
}

\author{
V. Z. Kresin \\ Lawrence Berkeley Laboratory, University of California, Berkeley, CA 94720 \\ E-mail: vzkresin@lbl.gov \\ Received June 19, 2001
}

\begin{abstract}
Josephson current flowing through an antiferromagnetic metallic barrier is studied. We consider the most interesting case of the spin-valve structure when the barrier is formed by ferromagnetic layers ordered antiferromagnetically in the direction perpendicular to the current. The most remarkable feature is an appearance of giant magnetooscillations of the current amplitude.
\end{abstract}

PACS: 74.50.+r, 74.80. Dm

\section{Introduction}

Study of various resonance phenomena is an important area of the condensed matter physics related to unique information about spectra and properties of various materials. The phenomena such as measurements of Shubnikov-de Haas oscillation, de Haas-van Alphen effects, cyclotron resonance, etc., are well-known and described in many textbooks and monographs.

In this article we will describe the effect of giant magnetooscillations. The proposed method which combines the Josephson tunneling spectroscopy and novel magnetic systems is very sensitive to magnetic structure of the compounds.

We focus on the special case of the $\mathrm{S}-\mathrm{N}-\mathrm{S}$ junctions when the normal $(\mathrm{N})$ metallic barrier is a magnetic compound. It is well known that the ferromagnetic material (F) used as a barrier frustrates the Josephson current because of the pairbreaking effect of the exchange field. Below we consider the antiferromagnetic barrier and, more specifically, the most interesting case of the socalled A-structure $\left(\mathrm{S}_{1}-\mathrm{A}-\mathrm{S}_{2}\right.$ junction $)$. This is the situation when the barrier is formed by multilayer system. It consists of ferromagnetic layers, perpendicular to the S-electrodes with an antiferromagnetic ordering in the direction perpendicular to the current. In other words, the neighboring layers have an opposite magnetization.
At first, we describe the compounds and their A-structure. Afterwards, we introduce the giant magnetooscillations phenomenon for the $\mathrm{S}_{1}-\mathrm{A}-\mathrm{S}_{2}$ junction.

\section{A-structure. Spin-valve effect}

There are two interesting systems which are characterized by A-structure. One of them is a mixed-valence manganite with some characteristic level of doping, and the second one is an artificial structure, so-called giant magnetoresistance (GMR) multilayer.

As is known, the most remarkable property of manganites (see review [1]) is the colossal magnetoresistance. These materials with the composition $\mathrm{A}_{1-x} \mathrm{Sr}_{x} \mathrm{MnO}_{3}(\mathrm{~A}=\mathrm{La}, \mathrm{Ca})$ have a rather complicated phase diagram.

The low temperature phase diagram has been analyzed in our papers [2,3]. The parent material, $\mathrm{LaMnO}_{3}$, is an insulator. The region $0.17<x<0.5$ corresponds to metallic state. The undoped manganite (e.g., $\mathrm{LaMnO}_{3}$ crystal) has the following cubic structure. The $\mathrm{Mn}^{3+}$ ions are located at the corners, and the La ion is at the center of the unit cell. In addition, the $\mathrm{Mn}^{3+}$ is caged by the $\mathrm{O}^{2-}$ octahedron; locally this forms an $\mathrm{MnO}_{6}$ complex with the $\mathrm{Mn}$ ion in the central position surrounded by light $\mathrm{O}$ ions. 
The $d$-shell of the Mn ion in the cubic environment is splitted into a doublet and triplet. It is important that the three-fold manifold $\left(t_{2 g}\right)$ is occupied by three $d$-electrons, whereas the upper double-degenerate term $e_{2 g}$ is occupied by one electron only.

The key ingredients (interactions) are the following: 1) the strong Hund's rule coupling (this is the largest energy scale) aligns all spins in the same direction; 2) hopping, and 3) cooperative JahnTeller (JT) effect. As a result, the total Hamiltonian is a sum:

$$
H=H_{H}+H_{t}+H_{J T} .
$$

Here

$$
\begin{gathered}
H_{H}=J_{H} \sum_{i} \sigma \mathbf{S}_{i}, \\
H_{t}=\sum_{i} t_{i, i+\delta}, \\
H_{J T}=\sum_{i} g\left(\tau_{i} \mathbf{Q}_{i}\right)+\sum_{i} J_{\mathrm{el}} \mathbf{Q}_{i}^{2},
\end{gathered}
$$

where $\mathbf{Q}$ is a normal coordinate, $\boldsymbol{\sigma}$ is the electronic spin, $\mathbf{S}$ is the ionic spin, $J_{\text {el }}$ is the elastic parameter, $\tau$ is the pseudospin.

The Hund's coupling between the local spin $\mathbf{S}$ $(S=3 / 2)$ formed by the $t_{2 g}$ electrons and the $e_{2 g}$ electron is described by the term (2); $\sigma$ (Pauli matrices) correspond to the spin of the $e_{2 g}$ electron; $J_{H} \sim 1 \mathrm{eV}$. Note, that the unit cell contains one $e_{2 g}$ electron and its motion through the lattice is described (in the tight-binding picture) by the term (3).

The third term in Eq. (1) describes another important ingredient which also affects the behavior of the system, namely the Jahn-Teller instability. Indeed, the $e_{2 g}$ electron is in the double-degenerate state, and it follows from the JT theorem, that the electron-lattice couplng will lead to a static distortion and consequently, to a change in the crystal symmetry. In Eq. (4) $g$ is the electron-phonon coupling constant, $\mathbf{Q}_{i}$ are the local active JT modes.

In a simple band picture with one electron per unit cell, the system should be metallic. This is contrary to the experimental fact that the parent compound is an insulator. Nevertheless, a more careful analysis which includes not only the hopping term, but also a strong Hund's interaction along with the cooperative Jahn-Teller effect, leads one to the picture of a peculiar band insulator $[2,3]$.

As noted above, Hund's coupling corresponds to the largest energy scale $\left(J_{H} \sim 1 \mathrm{eV}\right)$, so that $J_{H}>>t, g Q_{0} ; t \sim g Q_{0} \sim 0.1 \mathrm{eV}$.

It is interesting that the magnetic structure of the undoped crystal belong to the A-type: antiferromagnetic ( $\mathrm{AF}$ ) ordering along the $Z$-axis along with the ferromagnetic ordering in the $X Y$ plane. The appearance of such a structure is caused by an interplay between the Hund's interaction and the hopping energy. In other words, the A-structure is stabilized by the gain in the kinetic energy of the band electron. One can explain the underlying physics, if we consider the two-center problem $\left(\mathrm{Mn}^{3+}\right.$ $\mathrm{Mn}^{4+}$ with spins $S_{1}$ and $S_{2}$ ). The low energy electronic term has a form:

$$
E=-J_{H} S+t \cos q / 2-O\left(t^{2} / J_{H}\right) .
$$

Therefore, at $J_{H}>>t$, the main gain in the energy is due to the $-J_{H} S$ term. Although, because of the averaging, there is no linear contribution to the total energy, a small gain, of order of $t^{2} / J_{H}$ is achieved. The equation $t^{2} / J_{H}<t$ sets a lower energy scale for the Neel temperature $T_{N}$ which is indeed small $(\sim 140 \mathrm{~K})$ relative to the structural transition temperature $(\sim 0.1 \mathrm{eV})$. Within a single layer the electrons may be treated as ferromagnetically polarized. This ferromagnetism is provided by the double-exchange mechanism introduced by Zener [4] and developed in [5,6].

As was noted above, the pure $\mathrm{LaMnO}_{3}$ compound can be treated as a band insulator. This means that a change in the carrier concentration might lead to metallic behavior.

Consider now a doped manganite, e.g., $\mathrm{La}_{1-x} \mathrm{Ca}_{x} \mathrm{MnO}_{3}$. An increase in $x$ will lead initially to the formation of finite clusters; each of them contains itinerant polarized electrons. Eventually one can observe a formation of the infinite cluster and the transition to the metallic ferromagnetic state. Such transition can be described by percolation theory.

A very interesting observation has been reported in [7-10]. The presence of metallic A-phase has been observed. The examples are the compounds: $\mathrm{A}_{1-x} \mathrm{Sr}_{x} \mathrm{MnO}_{3} \quad(\mathrm{~A}=\mathrm{La}, \mathrm{Nd})$ with $x=0.55$ or $\mathrm{Pr}_{0.5} \mathrm{Sr}_{0.5} \mathrm{MnO}_{3}$. This is a natural spin-valve structure.

Another case is an artificial A-structure which is a basic for the GMR phenomenon (see reviews [11,12]). Such multilayer system (e.g., $\mathrm{Co}-\mathrm{Al}_{2} \mathrm{O}_{3}-\mathrm{Co}$ ) consists of alternating ferromagnetic and nonmagnetic la- 
yers with an antiferromagnetic ordering in the direction perpendicular to the layers.

Let us evaluate the energy spectrum for such metallic A-system. This is an important step for the calculation of the Josephson current through such a barrier. For concrettness, we consider the metallic manganite in A-phase.

The Hamiltonian for the barrier's states has a form:

$$
\begin{array}{rl}
H=\sum_{\mathbf{p}} & t(\mathbf{p}) a_{\mathbf{p} \sigma^{+}}^{+} a_{\mathbf{p} \sigma^{\prime}}^{+}+\sum_{\mathbf{p}, \mathbf{Q}} J_{H} S(\mathbf{Q}) a_{\mathbf{p} \sigma^{\prime}}^{+}\left(\hat{\sigma}_{z}\right)_{\sigma^{\prime} \sigma^{\prime \prime}} a_{\mathbf{p}-\mathbf{Q} ; \sigma^{\prime \prime}}+ \\
+ & \sum_{\mathbf{p}, \mathbf{Q}} J_{H} S(-\mathbf{Q}) a_{\mathbf{p} \sigma^{\prime}}^{+}\left(\hat{\sigma}_{z}\right)_{\sigma^{\prime} \sigma^{\prime \prime}} a_{\mathbf{p}-\mathbf{Q} ; \sigma^{\prime \prime}}+ \\
& +\sum_{\mathbf{p}} J_{H} M a_{\mathbf{p} \sigma^{\prime}}^{+}\left(\hat{\sigma}_{x}\right)_{\sigma^{\prime} \sigma^{\prime \prime}} a_{\mathbf{p}-\mathbf{Q} ; \sigma^{\prime \prime}}
\end{array}
$$

Here $t=t_{\perp}+t_{\|}, t_{\perp}$ and $t_{\|}$are electron hopping parameters for the in-plane and out-of plane motion, $J_{H}$ is the Hund's coupling, and $S(\mathbf{Q})$ is the Fourier component of the AFM ordering along the $c$ directions; $\left\langle S_{z}\right\rangle=S(-1)^{n}$. The structural vector $\mathbf{Q}=(0,0, \pi / a)$ reduces the Brillouin zone ( $a$ is the lattice constant). We consider a more general case of a canted structure; $M$ is the canted magnetic moment: $S_{i}=\left( \pm\left\langle S_{z}\right\rangle, M_{x}\right), S_{z}^{2}+M_{x}^{2}=S(S+1) \cong S^{2}$. Note that the Jahn-Teller effect is not essential for the metallic state.

With use of equation of motion

$$
\left(\tilde{\varepsilon}-t_{\|}\right) a_{\mathbf{k} \sigma}=J_{H} S( \pm \mathbf{Q}) \hat{\sigma}_{z} a_{\mathbf{k}-\mathbf{Q} ; \sigma}+J_{H} M\left(\hat{\sigma}_{x}\right)_{\sigma \sigma^{\prime}} a_{\mathbf{k} \sigma^{\prime}}
$$

and similar equation for $\mathbf{k} \rightarrow \mathbf{k}+\mathbf{Q}\left(\tilde{\varepsilon}=\varepsilon-t_{\perp}\right)$, one can determine the following energy spectrum:

$$
\tilde{\varepsilon}= \pm\left[J_{H}^{2} S^{2} \pm 2 J_{H} M t_{\|}+t_{\|}^{2}\right]^{1 / 2}
$$

which consists of four branches. For the manganites $J_{H}>>t$ (see above). As a result, for these systems, only two branches are filled:

$$
\tilde{\varepsilon}_{1,2} \cong-J_{H} S \pm(M / S) t_{\|}
$$

\section{Josephson current through an antiferromagnetic barrier. Magnetooscillations}

Let us evaluate the Josephson current through the A-barrier. We consider the case of a singlet pairing ( $s$ or $d$ ), that is the Cooper pair consists of two carriers with opposite spins and momenta. The A-barrier contains the states with opposite spins; they belong to the neighboring layers and, contrary to the $\mathrm{F}$ barrier, one should not expect any frustration for the Josephson current. Nevertheless, the calculation of the current should be carried out with a considerable care. Indeed, the Josephson current is a transfer of correlated electrons and this implies that the layers are not totally independent. The barrier should be treated as an anisotropic metal with the spectrum (8), (9). The Josephson current can be evaluated with use of the spectrum (8), (9) and the interface Hamiltonian:

$$
H_{\text {pair }}=V \Delta_{i} \Psi(i) \Psi^{+}(i)
$$

which described the transition of pair in the $i$ th superconductor $(i=1,2)$ into the barrier $\left(\Delta_{1}, \Delta_{2}\right.$ are the order parameters, $\Psi(i)$ and $\Psi^{+}(i)$ are the field operators for electrons of the barrier). With the use of Eq. (10) we find a correction to the thermodynamic potential, $\delta \Omega$, caused by the barrier; the current is obtained as $\delta \Omega / \delta \varphi$ with $\varphi$ being a phase difference between two superconductors' order parameters. The Josephson current is determined by the Cooper diagram:

$$
K=\pi T\left|V^{2}\right| \sum_{\omega_{n}} \Delta_{1} \Delta_{2} \int d \mathbf{p} d \mathbf{q} \exp (i \mathbf{q} \mathbf{L}) \Pi\left(i \omega_{n}, \mathbf{q}\right)
$$

where

$$
\begin{aligned}
& \Pi\left(i \omega_{n}, \mathbf{q}\right)=\sum_{\sigma, \sigma^{\prime}, \sigma^{\prime \prime}, \sigma^{\prime \prime \prime}}\left(\hat{\sigma}_{y}\right)_{\sigma, \sigma^{\prime}} G_{\sigma, \sigma^{\prime}}\left(i \omega_{n}, \mathbf{p}\right)\left(\sigma_{y}^{\prime}\right)_{\sigma^{\prime \prime}, \sigma^{\prime \prime \prime}} \times \\
& \times G_{\sigma^{\prime}, \sigma^{\prime \prime \prime}}\left(-i \omega_{n}, \mathbf{q}-\mathbf{p}\right)=2\left[G_{\downarrow \uparrow}\left(i \omega_{n}, \mathbf{p}\right) \times\right. \\
& \left.\times G_{\uparrow \downarrow}\left(-i \omega_{n}, \mathbf{q}-\mathbf{p}\right)-G_{\downarrow \downarrow}\left(i \omega_{n}, \mathbf{p}\right) G_{\uparrow \uparrow}\left(-i \omega_{n}, \mathbf{q}-\mathbf{p}\right)\right]
\end{aligned}
$$

(we use the method of thermodynamic Green's functions, see, e.g., [13]), $L$ is the distance between the superconductors.

The Green's function entering Eq. (12) can be expressed in terms of new Fermi amplitudes which correspond to the branches (8), (9). The transformation to the new amplitudes can be described by canonical transformation $a_{\mathbf{p} \sigma}=\sum_{i} K_{\sigma i} \alpha_{i \mathbf{p}} ;$ one can show that $\sigma \equiv(\uparrow \downarrow), K_{\sigma 1}=0.5[1-(M / S)]^{1 / 2} ; K_{\uparrow 2}=$ $=-K_{\downarrow 2}=0.5[1+(M / S)]^{1 / 2}$, where the operators $\alpha_{i \mathbf{p}}$ correspond to the branches (8), (9). Then we obtain the following expression for the Cooper term: 


$$
\begin{gathered}
j_{m}=\left(1-\frac{M}{S}\right)\left|V^{2} \| \Delta_{1}\right|^{2}\left|\Delta_{2}\right|^{2} \pi T \times \\
\times \sum_{\omega_{n}>0} \int d l v_{\perp} \int d p_{z} \exp \left(-\frac{\omega_{n} L}{v_{\perp}}\right) \cos \left(\frac{L t_{\|} M}{S v_{\perp}}\right) .
\end{gathered}
$$

In the tight-binding approximation $t_{\|}=t_{0} \cos \left(p_{z} d_{c}\right)$. Equation (13) contains the integration over the cross-section of the Fermi surface $(d l)$.

As a result, we arrive at the following expression for the amplitude of the Josephson current:

$$
J_{m}=r\left[1-(M / S)^{2}\right] f\left(L / \zeta_{N}\right) J_{0}(\beta M / S) .
$$

Here $\zeta_{N}=h v_{F} / 2 \pi T$ is the coherence length inside the barrier, $\beta=\left(t_{0} / T_{c}\right)\left(L / \xi_{0}\right) ; \xi_{0}=h v_{F} / 2 \pi T_{c}, v_{F}=$ $=v_{F}^{0}$ is the minimum value of the component of the Fermi velocity along $L, r \propto p \Delta_{1} \Delta_{2} V^{2}, f(x)=\exp (-x)$ for $x>>1$ and $f(x)=x^{-1}(x<<1)$. Therefore, near $T=0 \mathrm{~K}$, the function $f=\zeta_{N} / L$. Note, that since $r \propto T$, the amplitude $J_{m}$ in this case does not depend on $T$. However, near $T_{c}$ and in the intermediate temperature region, the exponential dependence $f=\exp \left(-L / \zeta_{N}\right)$ is perfectly realistic.

\section{Discussion}

Consider the junction placed in an external magnetic field. Its presence creates the canting. For weak magnetic field the canting is small and the dependence of the amplitude is determined by the factor $J_{0}(\beta M / S)$ in Eq. (14). Since $\beta>>1$, one can use an asymptotic expression for the Bessel function, and we obtain:

$$
\begin{gathered}
J_{m}=r\left[1-(M / S)^{2}\right] \times \\
\times \exp \left(-\frac{L}{\xi_{N}(T)}\right)\left(\frac{\pi \beta M}{2 S}\right)^{-1 / 2} \cos \left(\frac{\beta M}{S}-\frac{\pi}{4}\right) .
\end{gathered}
$$

The expression is valid if $L \gg \zeta_{N}$.

Equations (14), (15) display remarkable property of the junction with an A-barrier, namely a phenomenon of giant magnetooscillations (GMO). Indeed, one can directly from Eq. (15) that the amplitude oscillates as a function of $M$, and, therefore, as a function of the external field $H$.

The GMO effect is caused by magnetic structure (spin-valve effect) and is entirely different from the usual Fraunhofer oscillations. Note also that, because $\beta>>1$, the period of GMO is much smaller. Moreover, one can choose such geometry of the Josephson contact that the only magnetic structure is affected by magnetic field. The increase in magnetic field leads eventually to the situation when $M=S$; then $J_{m}=0$ (see Eqs. (14), (15)). This occurs because the external field affect magnetic ordering in the barrier. If $M=S$, then we are dealing with 3D ferromagnetic ordering. As for the the $\mathrm{S}-\mathrm{F}$ contact, it frustrates the Josephson current; this is due to the pair-breaking effect of the exchange field (we do not consider some modifications caused by the spin-orbital coupling, see [14]). Therefore, the amplitude of the current can be controlled by an external magnetic field and even switched off at larger $H$ (order of $1 \mathrm{~T}$ ). Note that for the GMR structure the switching can be obtained with use of weaker magnetic field (order of 3-4 Oe).

In summary, we show that the Josephson current can flow through an antiferromagnetic barrier. The most interesting case corresponds to the case of the A-ordering in the direction perpendicular to the current. One can show that the amplitude of the current oscillates as a function of applied magnetic field (GMO).

Lev Vasiljevich Shubnikov was a remarkable scientist who made key contributions to various areas of physics. We are pleased that this paper is published in the Special Issue devoted his memory.

1. J. Coey, M. Viret, and S. von Molnar, Adv. Phys. 48, 167 (1999).

2. L. Gor'kov and V. Kresin, JETP Lett. 67, 985 (1998).

3. M. Dzero, L. Gor'kov, and V. Kresin, Eur. Phys. J. B14, 459 (2000).

4. G. Zener, Phys. Rev. 82, 403 (1951).

5. P. Anderson and H. Hasegawa, Phys. Rev. 100, 675 (1955).

6. P. de Gennes, Phys. Rev. 118, 141 (1960).

7. H. Kawano, R. Kajimoro, H. Yoshizawa, Y. Tomioka, H. Kuwahara, and Y. Tokura, Phys. Rev. Lett. 78, 44253 (1997)

8. H. Fujisturo, M. Ikebe, and Y. Konno, J. Phys. Soc. Jpn. 67, 1799 (1998)

9. M. Isumi, T. Manako, Y. Konishi, M. Kawasaki, and Y. Tokura, Phys. Rev. B61, 12187 (2000).

10. Y. Morimoto, T. Akimoto, A. Nakamura, K. Ohoyama, and M. Ohashi, Phys. Rev. B58, 5544 (1998).

11. S. S. P. Parkin, Annu. Rev. Mater. Sci. 25, 358 (1995).

12. Ultrathin Magnetic Structures II, B. Heinrich and J. A. C. Bland (eds.), Springer-Verlag, Berlin (1994).

13. A. Abrikosov, L. Gor'kov, and I. Dzyaloshinskii, Methods of Quantum Field Theory in Statistictical Physics, Dover, NY (1975)

14. E. Demler, G. Arnold, and M. Beasley, Phys. Rev. B55, 15174 (1997). 